\title{
ALEKSANDR ALEKSANDROVICH NIKOLSKII: MARKING THE 100TH ANNIVERSARY OF HIS BIRTH
}

\section{G.G. Sudakov}

Central Aerohydrodynamic Institute (TsAGI), 1, Zhukovsky Str., Zhukovsky, Moscow Region, 140180,Russian Federation, E-mail: uzts@tsagi.ru

This paper is dedicated to the 100th anniversary of Aleksandr Aleksandrovich Nikolskii.

KEY WORDS: Nikolskii, TsAGI, mechanics, aerodynamics, anniversary

February 13, 2019, marks the 100th anniversary of the birth of Aleksandr Aleksandrovich Nikolskii (Fig. 1), a great scientist in mechanics and aerodynamics. In 1941, A.A. Nikolskii graduated from the Department of Mathematics and Mechanics at Moscow State University and was invited to work at TsAGI. Thus, for many years his scientific and pedagogical activities were closely associated with TsAGI.

A.A. Nikolskii's main areas of scientific activity and interest were the following:

- Transonic and supersonic ideal gas flows;

- Fluid and gas flows with separation;

- Physical fluid dynamics, namely, magnetic hydrodynamics, hypersonic radiation, and rarefied gases;

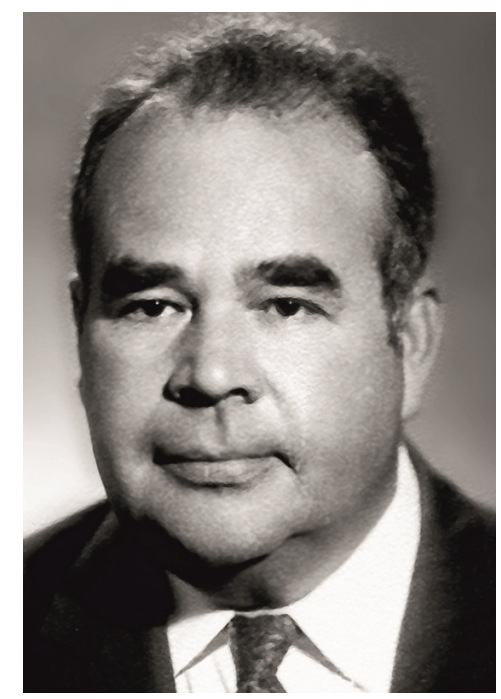

FIG. 1: Alexandr Alexandrovich Nikolskii 
- Applied gas dynamics, namely, gas flows with perforated boundaries, critical modes of supersonic ejectors, and destruction waves of gas-saturated rocks; and

- Mechanics of rotating fluids.

In his younger years, A.A. Nikolskii had already accomplished a series of fundamental theoretical investigations in the field of transonic and supersonic gas flows, which formed the basis for his doctoral dissertation (1949). The widely known Nikolsky-Taganov theorem is among these results. In subsequent years, A.A. Nikolskii was given awards several times:

- 1949: First prize and gold medal named after professor N.E. Zhukovsky;

- 1950: Order of the Red Banner of Labor; and

- 1951: State Prize.

Aleksandr Aleksandrovich Nikolskii was distinguished by the breadth and depth of his scientific research and a special sense for exact analytical results. He found:

- The necessary conditions for a shock-free transition through the speed of sound;

- Exact solutions for supersonic spatial flows, including conical axially symmetric rarefaction flow that complements Busemann's solutions;

- The shape of a body of revolution with a duct of minimal wave drag;

- Similarity laws for a flow with separation over low-aspect-ratio wings;

- The effect of flow separation from the side edges of a finite wing on the lift force;

- Exact solutions for the unsteady separation of the flow over two-dimensional lift surfaces;

- Boundary conditions on the walls with longitudinal and transverse slit perforations;

- The effect of the perforation coefficient and Mach number on the degree of the induction effect of a wall;

- Exact solutions to the Boltzmann equation for rarefied gas;

- New results in the theory of magnetohydrodynamic motion of an ideal fluid;

- Laws of motion of rotating fluid; and

- A mathematical model of the wave destruction of gas-saturated rocks.

The fundamental results achieved by A.A. Nikolskii were a major milestone in the development of new directions in science. They were used in practice in the development of aircraft and experimental aerodynamic facilities.

The results of the scientific activities of A.A. Nikolskii found wide recognition in Russia and abroad. He was a member of the presidium of the International Union of Theoretical and Applied Mechanics; a member of the presidium of the Soviet Union National Committee on Mechanics; an associate editor-in-chief of the journal Izvestiya Akademii Nauk USSR Mekhanika Zhidkosti $i$ Gaza (Fluid Dynamics); a member of the editorial boards of other leading publications in mechanics; and a member of the scientific councils of TsAGI, the Institute of Mechanics of the 
Academy of Sciences of USSR, the Computing Center of the Academy of Sciences of USSR, and the Moscow Institute of Physics and Technology. A.A. Nikolskii was the initiator and the first editor-in-chief of the journal Uchenye Zapiski TsAGI.

As a professor at the Moscow Institute of Physics and Technology, Aleksandr Aleksandrovich Nikolskii paid much attention to the education of young scientists. Starting in 1969, he held the following special courses in the Department of Aeromechanics and Flight Engineering: "Separated Flows of an Ideal Fluid" (a completely original course, based on his own research) and "Theory of Hypersonic Flows."

Among his students were the following graduates of the Moscow Institute of Physics and Technology and Moscow State University: S.K. Betyayev, V.F. Molchanov, A.G. Zarubin, G.G. Soudakov, S.B. Zakharov, A.V. Zubtsov, A.V. Voevodin, and A.M. Gaifullin. All of these former students of A.A. Nikolskii are doctors and candidates of science who form the core of the scientific school of A.A. Nikolskii, and honorably continue his work and traditions.

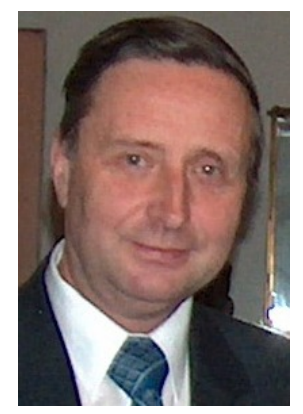

Georgii Grigorievich Sudakov, Doctor in Technical Sciences, Chief Researcher, TsAGI

Volume 50, Issue 1, 2019 